\title{
The Comparison of Two Error Estimates for Approximate Solutions of the Poisson Equation
}

\author{
G. Lüttgens
}

\begin{abstract}
The purpose of the present paper is to discuss different error estimates for the numerical solution of a Dirichlet problem for the Poisson equation, calculated via the five point (discrete) Laplacian. Whereas the first error bound, a sum of ordinary $L^{\infty}$-moduli, is deduced from the usual stability inequality, use is made of some properties of the discrete Green function to verify another stability inequality in terms of $l^{1}$-norms, which then implies the second estimate via $\tau$-moduli multiplied by a logarithm factor. In the following we will show that it depends on the solution of the boundary value problem which measure of smoothness or rather which error estimate delivers the correct rate of convergence. The paper concludes with some remarks illustrating relations to the well-known logarithm factor in connection with finite element approximation.
\end{abstract}

Keywords: Poisson equation, finite difference method, stability, comparison of error bounds AMS subject classiflcation: $35 \mathrm{~J} 05,41 \mathrm{~A} 25,65 \mathrm{~N} 12$

\section{Introduction}

Consider the Dirichlet problem for the Poisson equation

$$
\begin{aligned}
\Delta u(x, y) & :=\frac{\partial^{2}}{\partial x^{2}} u(x, y)+\frac{\partial^{2}}{\partial y^{2}} u(x, y)=\varphi(x, y) & & ((x, y) \in \Omega) \\
u(x, y) & =\psi(x, y) & & ((x, y) \in \Gamma)
\end{aligned}
$$

on the open unit square $\Omega:=\{(x, y): 0<x, y<1\} \subset \mathbb{R}^{2}$ (with $\mathbb{R}$ being the real axis) and $\Gamma:=\partial \Omega$, the boundary of $\Omega$. Throughout it will be assumed that $\varphi$ and $\psi$ are continuous, real-valued functions on $\Omega$ and $\Gamma$, respectively. Furthermore, we will restrict the following treatment to those problems (1) for which the solution $u$ exists and belongs to the Banach space $C^{2}(\bar{\Omega})$ of all real-valued functions $u$ on $\bar{\Omega}:=\Omega \cup \Gamma$ which possess continuous partial derivatives $\left(\frac{\partial}{\partial x}\right)^{i}\left(\frac{\partial}{\partial y}\right)^{j} u(x, y)$ for $0 \leq i \leq i+j \leq 2$ such that the norm

$$
\|u\|_{C^{2}(\bar{\Omega})}:=\sum_{0 \leq i \leq i+j \leq 2}\left\|\left(\frac{\partial}{\partial x}\right)^{i}\left(\frac{\partial}{\partial y}\right)^{j} u\right\|_{C(\bar{\Omega})}
$$

G. Lüttgens: Rhein.-Westf. Techn. Hochschule, Lehrstuhl A für Mathematik, D - 52056 Aachen 
is finite. Here $C(\bar{\Omega})$ is the space of all continuous functions equipped with the usual sup-norm $\|u\|_{C(\bar{\Omega})}:=\sup \{|u(x, y)|:(x, y) \in \bar{\Omega}\}$.

Let $h=\frac{1}{n}$ with $n \in \mathbb{N}$, the set of natural numbers. An approximate solution $u_{h}$, defined on the uniform grid

$$
\begin{aligned}
& \bar{\Omega}_{h}:=\left\{(x, y): x=\frac{i}{n} \text { and } y=\frac{j}{n}(0 \leq i, j \leq n)\right\} \\
& \Omega_{h}:=\bar{\Omega}_{h} \cap \Omega \quad \text { and } \quad \Gamma_{h}:=\bar{\Omega}_{h} \cap \Gamma
\end{aligned}
$$

is obtained as the unique solution of the discrete counterpart to problem (1)

$$
\begin{aligned}
\Delta_{h} u(x, y) & =\varphi(x, y) & & \left((x, y) \in \Omega_{h}\right) \\
u(x, y) & =\psi(x, y) & & \left((x, y) \in \Gamma_{h}\right) .
\end{aligned}
$$

Thereby the five point Laplacian $\Delta_{h}$ (cf. [8: p. 45] and [12: p. 49]) is given by

$$
\Delta_{h} u_{h}(x, y):=\frac{1}{h^{2}}\left[\Delta_{h}^{2,0} u_{h}(x, y)+\Delta_{h}^{0,2} u_{h}(x, y)\right],
$$

i.e., the partial derivatives of the Laplacian $\Delta$ are now replaced by symmetric differences $\Delta_{h}^{2,0} u_{h}(x, y):=u_{h}(x+h, y)-2 u_{h}(x, y)+u_{h}(x-h, y)$ and $\Delta_{h}^{0,2} u_{h}(x, y)$, defined analogously.

The aim of this paper is to discuss and to compare two different estimates for the, approximation error

$$
\left\|u-u_{h}\right\|_{\bar{\Omega}_{h}}:=\sup _{(x, y) \in \bar{\Omega}_{h}}\left|u(x, y)-u_{h}(x, y)\right|
$$

given in terms of moduli of smoothness for partial derivatives of the exact solution $u$ (cf. (6) and (11)). Besides the ordinary $L^{\infty}$-modulus

$$
\omega_{2,0}(\delta, u):=\sup \left\{\left|\Delta_{h}^{2,0} u(x, y)\right|:|h| \leq \delta \text { and }(x \pm h, y) \in \bar{\Omega}\right\}
$$

( $u \in C(\bar{\Omega}) ; \omega_{0,2}(\delta, u)$ analogously) we want to study a new kind of measure of smoothness, the so-called $\tau$-modulus, introduced by P. P. Korovkin and Bl. Sendov independently around 1968, which has turned out to be a powerful tool for various applications in approximation theory and numerical analysis (see [14]). This $L^{\mathbf{1}}$-modulus is defined as the integral (e.g.)

$$
\tau_{2,0}(\delta, u):=\int_{\tilde{\Omega}} \omega_{2,0}(\delta, u ;(x, y)) d(x, y) \quad(u \in C(\Omega))
$$

over the local modulus of continuity

$$
\begin{aligned}
\omega_{2,0}(\delta, u ;(x, y)):=\sup \{ & \left|\Delta_{h}^{2,0} u(\bar{x}, \bar{y})\right|: \\
& (\bar{x} \pm h, \bar{y}) \in([x-\delta, x+\delta] \times[y-\delta, y+\delta]) \cap \bar{\Omega}\} .
\end{aligned}
$$

For similar (comparison) considerations in connection with quadrature formulas or ordinary differential equations we refer to $[2,3,14]$. 


\section{The error estimates}

Using the well-known stability inequality (cf. [10: p. 464])

$$
\left\|v_{h}\right\|_{\bar{\Omega}_{h}} \leq \frac{1}{2}\left\|\Delta_{h} v_{h}\right\|_{\Omega_{h}}+\left\|v_{h}\right\|_{\Gamma_{h}}
$$

in $l^{\infty}$-norms (cf. (3)) for $v_{h}=u-u_{h}$ and the representation of the truncation error

$$
\begin{aligned}
\tau_{h} u(x, y) & :=\Delta_{h}\left(u-u_{h}\right)(x, y) \\
& =\frac{-1}{h^{2}} \int_{0}^{h}(h-s)\left[\Delta_{g}^{2,0} u_{x x}(x, y)+\Delta_{g}^{0,2} u_{y y}(x, y)\right] d s
\end{aligned}
$$

in terms of the solution $u$ of problem (1), one obtains (cf. [1])

$$
\left\|u-u_{h}\right\|_{\bar{\Omega}_{h}} \leq \frac{1}{4} \omega^{*}(h, u):=\frac{1}{4}\left[\omega_{2,0}\left(h, u_{x x}\right)+\omega_{0,2}\left(h, u_{y y}\right)\right] \quad\left(u \in C^{2}(\bar{\Omega})\right) .
$$

Thereby and in the following let $u_{h}$ be the solution of the discrete problem (2) corresponding to the data $\varphi(x, y)=\Delta u(x, y)$ and $\psi(x, y)=u(x, y)$ for $(x, y) \in \Omega$ and $\Gamma$, respectively. The fact that the estimate (6) is sharp with regard to the rate of convergence has been established in connection with general Lipschitz classes, determined by abstract moduli of continuity, i.e., by functions $\omega$ (e.g., $\omega(t)=t^{\alpha}, 0<\alpha^{*} \leq 1$ ), continuous on $[0, \infty)$ and with

$$
0=\omega(0)<\omega(s) \leq \omega(s+t) \leq \omega(s)+\omega(t) \quad(s, t>0)
$$

Indeed, for every abstract modulus of continuity there exists a counterexample $u_{\omega} \in$ $C^{2}(\bar{\Omega})$ such that

$$
\omega^{*}\left(\delta, u_{\omega}\right)=\mathcal{O}\left(\omega\left(\delta^{2}\right)\right),
$$

thus $\left\|u_{\omega}-u_{\omega, h}\right\|_{\bar{\Omega}_{h}}=\mathcal{O}\left(\omega\left(h^{2}\right)\right)$, but on the other hand

$$
\left\|u_{\omega}-u_{\omega, h}\right\|_{\Omega_{h}} \neq o\left(\omega\left(h^{2}\right)\right)
$$

A proof of (7) and (8) is given in [1] via an application of a quantitative extension of the uniform boundedness principle (cf. [5] and the literature cited there). Additionally use is made of some properties of the discrete Green function $G_{h}(\xi, \eta)$ on $(\xi, \eta) \in \bar{\Omega}_{h} \times \Omega_{h}$, defined for fixed $\eta \in \Omega_{h}$ as the unique solution of the problem

$$
\begin{array}{rlrl}
\left(\Delta_{h} G_{h}(\cdot, \eta)\right)(\xi) & =\left\{\begin{array}{lll}
h^{-2} & \text { if } \xi=\eta \\
0 & \text { if } \xi \neq \eta
\end{array}\right. \\
G_{h}(\xi, \eta) & =0 & & \left(\xi \in \Omega_{h}\right)
\end{array}
$$

In particular the Green function is non-positive for all $(\xi, \eta) \in \bar{\Omega}_{h} \times \Omega_{h}$, and there holds true the identity (cf. [8: p. 58])

$$
v_{h}(\xi)=h^{2} \sum_{\eta \in \Omega_{h}} G_{h}(\xi, \eta) \Delta_{h} v_{h}(\eta) \quad\left(\xi \in \bar{\Omega}_{h}\right)
$$


for grid functions $v_{h}$ (i.e., real-valued functions defined on the grid $\bar{\Omega}_{h}$ ) which fulfill the condition with $v_{h}\left(\Gamma_{h}\right)=\{0\}$.

Let us now develop the second error estimate announced. In view of equation (9) there follows a further stability inequality ( $v_{h}$ a grid function with $v_{h}\left(\Gamma_{h}\right)=\{0\}$ )

$$
\left\|v_{h}\right\|_{\bar{\Omega}_{\Lambda}} \leq\left\|G_{h}\right\|_{\bar{\Omega}_{h} \times \Omega_{\Lambda}} h^{2} \sum_{\eta \in \Omega_{h}}\left|\Delta_{h} v_{h}(\eta)\right|
$$

with an $l^{1}$-norm at the right-hand side, similar to that used for ordinary differential equations in, e.g., $[2,6]$. In this connection it is of great importance to estimate the $l^{\infty}$-norm of the Green function $\left\|G_{h}\right\|_{\tilde{\Omega}_{h} \times \Omega_{h}}:=\sup \left\{\left|G_{h}(\xi, \eta)\right|:(\xi, \eta) \in \bar{\Omega}_{h} \times \Omega_{h}\right\}$.

Lemma 1: Let $\eta^{*}:=\left(\frac{1}{2}, \frac{1}{2}\right)$ and $n=\frac{1}{h} \in \mathbb{N}$ be even. Then there exist constants $M_{1}, M_{2}>0$ such that

$$
M_{1}|\log h| \leq-G_{h}\left(\eta^{*}, \eta^{*}\right) \leq\left\|G_{h}\right\|_{\Omega_{h} \times \Omega_{h}} \leq M_{2}|\log h|
$$

The last inequality holds true for odd $n \geq 2$, too.

In [9] the lower estimate is proved for a similar discretisation with the help of orthogonal eigenfunctions of the operator inverse to $\Delta_{h}$. In [11: p. 125] the present situation is dealt with. For the upper bound see also [8: p. 59].

Lemma 2: Let $n=\frac{1}{h} \geq 2$ and $v_{h}$ be a grid function with $v_{h}\left(\Gamma_{h}\right)=\{0\}$.

(a) There holds the stability inequality $\left(M_{3}<\infty\right)$

$$
\left\|v_{h}\right\|_{\bar{\Omega}_{h}} \leq M_{3}|\log h|\left(h^{2} \sum_{\eta \in \Omega_{h}}\left|\Delta_{h} v_{h}\right|\right) .
$$

(b) The factor $|\log h|$ on the right-hand side cannot be improved to o( $|\log h|)$.

Proof: The first assertion follows by (10) and Lemma 1 . Choosing $v_{h}(\xi):=$ $G_{h}\left(\xi, \eta^{*}\right)$ for $\eta^{*}=\left(\frac{1}{2}, \frac{1}{2}\right)$ and $n$ even one obtains

$$
\frac{\left\|G_{h}\left(\cdot, \eta^{*}\right)\right\|_{\bar{\Omega}_{h}}}{h^{2} \sum_{\eta \in \Omega_{h}}\left|\Delta_{h} G_{h}\left(\eta, \eta^{*}\right)\right|} \geq \frac{\left|G_{h}\left(\eta^{*}, \eta^{*}\right)\right|}{h^{2} h^{-2}} \geq M_{1}|\log h|
$$

which already completes the proof

Replacing the $l^{1}$-norm by an integral there results the following estimate in terms of the $\tau$-modulus (cf. (6)):

$$
\tau^{*}(\delta, u):=\tau_{2,0}\left(\delta, u_{x x}\right)+\tau_{0,2}\left(\delta, u_{y y}\right) \quad\left(u \in C^{2}(\bar{\Omega}), \delta>0\right) .
$$

Theorem 3: Let $u$ be the solution of problem (1) satisfying $u \in C^{2}(\bar{\Omega})$. Then for the solution $u_{h}$ of the discrete problem (2) the estimate

$$
\left\|u-u_{h}\right\|_{\Omega_{h}} \leq M_{4}|\log h| \tau^{*}(h, u)
$$


holds true $\left(n=\frac{1}{h} \geq 2\right)$. has

Proof: For $\eta:=\left(\eta_{1}, \eta_{2}\right) \in \Omega_{h}$ and $(x, y) \in A_{\eta}:=\left[\eta_{1}, \eta_{1}+h\right] \times\left[\eta_{2}, \eta_{2}+h\right] \cap \bar{\Omega}$ one

$$
h^{2} \omega_{2,0}\left(h, u_{x x} ; \eta\right) \leq \int_{A_{h}} \omega_{2,0}\left(2 h, u_{x x} ;(x, y)\right) d(x, y)
$$

since $\left[\eta_{1}-h, \eta_{1}+h\right] \times\left[\eta_{2}-h, \eta_{2}+h\right] \subset[x-2 h, x+2 h] \times[y-2 h, y+2 h]$ for all $(x, y) \in A_{\eta}$. In view of Lemma 2 and (5) it follows

$$
\begin{aligned}
\left\|u-u_{h}\right\|_{\Omega_{h}} & \leq M_{3}|\log h|\left(h^{2} \sum_{\eta \in \Omega_{h}}\left|\tau_{h} u(\eta)\right|\right) \\
& \leq M_{3}|\log h|\left(h^{2} \sum_{\eta \in \Omega_{h}}\left(\omega_{2,0}\left(h, u_{x x} ; \eta\right)+\omega_{0,2}\left(h, u_{y y} ; \eta\right)\right)\right) \\
& \leq M_{3}|\log h| \int_{\mathfrak{\Omega}}\left(\left(\omega_{2,0}\left(2 h, u_{x x} ;(x, y)\right)+\omega_{0,2}\left(2 h, u_{y y} ;(x, y)\right)\right) d(x, y) .\right.
\end{aligned}
$$

Finally the inequality $\tau_{2,0}\left(2 h, u_{x x}\right) \leq c \tau_{2,0}\left(h, u_{x x}\right)$ (cf. [14: p. 8] for the one-dimensional case or [11: p. 32]) delivers the assertion

Notice that the inequality (11) is sharp in an "asymptotic" sense. Due to Theorem $4 c)$ there exist $g_{n} \in C^{2}(\bar{\Omega})$ with

$$
\begin{gathered}
|\log h| \tau^{*}\left(h, g_{n}\right)=\mathcal{O}\left(|\log h| h^{2}\right) . \\
\left\|g_{n}-g_{n, h}\right\|_{\bar{\Omega}_{h}} \neq o\left(|\log h| h^{2}\right) .
\end{gathered}
$$

A result analogous to (12) follows for the error estimate (6) in terms of $\omega^{*}$, if one chooses $g_{n}=|\log h| h^{2} f_{\omega} / \omega\left(h^{2}\right)$ with $f_{\omega}$ in accordance with (7) and (8) for a fixed abstract modulus $\omega$.

\section{The comparison}

For problems, similar to (1) and (2) but in connection with quadrature formulas or ordinary differential equations, there are well-known error estimates in terms of $\boldsymbol{\omega}$ moduli as well as $\tau$-moduli ( $c f .[2,3,14]$ ). Contrary to the considerations there in the present situation none of the error bounds (6) and (11) majorizes the other one, since indeed on the one hand there holds true

$$
\tau^{*}(\delta, u) \leq \omega^{*}(\delta, u) \quad\left(u \in C^{2}(\bar{\Omega})\right)
$$

and for some special functions one even has $\tau^{*}(\delta, u) \leq \delta \omega^{*}(\delta, u)$ (cf. [7]). But on the other hand the inequality (11) additionally contains a logarithm factor. Really conferring the two estimates one gets the following result. 
Theorem 4: Depending on the solution $u$ of problem (1) each of the error bounds (6) and (11) partly yields the true rate of convergence.

a) For $z(x, y):=x^{4} \in C^{2}(\bar{\Omega})$ there holds true $\left(\delta \leq \frac{1}{2}\right)$

$$
\begin{aligned}
\omega^{*}(\delta, z) & =24 \delta^{2} \\
|\log \delta| \tau^{*}(\delta, z) & =\left(24 \delta^{2}-32 \delta^{3}\right)|\log \delta| \\
\left\|z-z_{h}\right\|_{\bar{\Omega}_{h}} & \neq o\left(h^{2}\right),
\end{aligned}
$$

i.e., the correct rate of convergence is only obtained via (6).

b) Let $f_{\alpha}(x, y):=x^{\alpha+2} /((\alpha+1)(\alpha+2))$ for $0<\alpha<1$. Then it follows

$$
\begin{aligned}
\omega^{*}\left(\delta, f_{\alpha}\right) & \geq\left(2-2^{\alpha}\right) \delta^{\alpha} \\
|\log \delta| \tau^{*}\left(\delta, f_{\alpha}\right) & \leq 33 \delta^{\alpha+1}|\log \delta| .
\end{aligned}
$$

In other words, using the inequality (11) instead of (6) the rate of convergence has been improved considerably.

c) There exists a sequence $\left(g_{n}\right)_{n=1}^{\infty} \subset C^{2}(\bar{\Omega})$ with $\left(h=\frac{1}{n}\right)$

$$
\begin{aligned}
\omega^{*}\left(h, g_{n}\right) & \neq o(1) \\
|\log h| \tau^{*}\left(h, g_{n}\right) & =\mathcal{O}\left(|\log h| h^{2}\right) \\
\left\|g_{n}-g_{n, h}\right\|_{\Omega_{h}} & \neq o\left(|\log h| h^{2}\right) .
\end{aligned}
$$

Therefore (11) delivers the correct "asymptotic" rate of convergence.

Proof: Via elementary calculations one has $\omega_{0,2}\left(\delta, z_{y y} ;(x, y)\right) \equiv 0$ and

$$
\omega_{2,0}\left(\delta, z_{x x} ;(x, y)\right)=\left\{\begin{array}{ll}
24 x^{2} & \text { for } x \in[0, \delta) \\
24 \delta^{2} & \text { for } x \in[\delta ; 1-\delta] \\
24(1-x)^{2}, & \text { for } x \in(1-\delta, 1]
\end{array} \quad(y \in[0,1])\right.
$$

hence $\tau^{*}(\delta, z)=24 \delta^{2}-32 \delta^{3}$. For the other two assertions of part a) see [1]. The statements in b) immediately follow from analogous one-dimensional results in [7].

Concerning statement $\mathrm{c}$ ), for the infinitely continuously differentiable function

$$
F(x):= \begin{cases}\exp \left(-1 /\left(1-x^{2}\right)\right) & \text { for }|x|<1 \\ 0 & \text { for }|x| \geq 1\end{cases}
$$

and $\eta^{*}=\left(\frac{1}{2}, \frac{1}{2}\right)$ consider

$$
g_{n}(\xi):=-\frac{1}{n^{2}} F\left(\frac{\xi_{1}-\frac{1}{2}}{\varepsilon_{n}}\right) F\left(\frac{\xi_{2}-\frac{1}{2}}{\varepsilon_{n}}\right) \quad\left(\xi=\left(\xi_{1}, \xi_{2}\right) \in \bar{\Omega}\right)
$$

with $\varepsilon_{n}:=\frac{2}{e \cdot n}$ and $n \in \mathbb{N}$ even. Obviously, the sequence $\left(g_{n}\right)_{n=1}^{\infty}$ is uniformly bounded in $C^{2}(\bar{\Omega})$, and for the support of $g_{n}$ one has the inclusion

$$
\operatorname{supp} g_{n} \subset\left[\frac{1}{2}-\varepsilon_{n}, \frac{1}{2}+\varepsilon_{n}\right]^{2} \text {. }
$$


Furthermore, there holds true $g_{n}\left(\eta^{*}\right)=-\frac{1}{e^{2} n^{2}}, g_{n}(\Gamma)=\{0\}$ and

$$
\Delta g_{n}(\xi)=\left\{\begin{array}{ll}
1 & \text { for } \xi=\eta^{*} \\
0 & \text { for } \xi \neq \eta^{*}
\end{array} \quad\left(\xi \in \Omega_{h}\right)\right.
$$

It follows that for the discrete approximate solution $g_{n, h}$ one indeed has $g_{n, h}(\xi)=$ $h^{2} G_{h}\left(\xi, \eta^{*}\right)$. so that by Lemma $1\left(n=\frac{1}{h}\right.$ even $)$

$$
\left\|g_{n}-g_{n, h}\right\|_{\hat{\Omega}_{h}} \geq\left|g_{n, h}\left(\eta^{*}\right)\right|-\left|g_{n}\left(\eta^{*}\right)\right| \geq M_{1}|\log h| h^{2}-\frac{h^{2}}{e^{2}} \neq o\left(|\log h| h^{2}\right),
$$

i.e., the last inequality of part c). Since (cf. (14))

$$
\omega^{*}\left(h, g_{n}\right) \geq \omega_{2,0}\left(h, g_{n, x x}\right) \geq\left|\Delta_{h}^{2,0} g_{n, x x}\left(\eta^{*}\right)\right|=1 \neq o(1)
$$

there only remains to establish the estimate of the $\tau$-modulus. In view of (13) we obtain $\omega_{2,0}\left(h, g_{n, x x} ;(x, y)\right)=\omega_{0,2}\left(h, g_{n, y y} ;(x, y)\right)=0$ if $\left|x-\frac{1}{2}\right| \geq h+\varepsilon_{n}$ or $\left|y-\frac{1}{2}\right| \geq h+\varepsilon_{n}$ and therefore

$$
\tau^{*}\left(h, g_{n}\right) \leq\left(h+\varepsilon_{n}\right)^{2} \omega^{*}\left(h, g_{n}\right) \leq c h^{2}\left\|g_{n}\right\|_{C^{2}(\Omega)}=\mathcal{O}\left(h^{2}\right) .
$$

Thus the proof of Theorem 4 is complete

Let us conclude with some remarks to finite element methods based upon piecewise linear functions. Considering the case $\psi \equiv 0$ and denoting the approximate solution by $\tilde{u}_{h}$, the rate of convergence of the error estimate (cf. [4: p. 172] and $[13] ; W^{2, \infty}(\bar{\Omega})$ the Sobolev space)

$$
\left\|u-\tilde{u}_{h}\right\|_{C(\Omega)}=\mathcal{O}\left(|\log h| h^{2}\right) \quad\left(u \in W^{2, \infty}(\tilde{\Omega})\right)
$$

cannot be improved to $o\left(|\log h| h^{2}\right)$. This assertion is established in [9] and in connection with general Lipschitz classes in [11: p. 185], each time with the help of the lower estimate for the norm of the Green function (cf. Lemma 1). Since the finite element approach is equivalent to the finite difference problem (cf. [4: p. 191] and [8: p. 160] for various triangulations)

$$
\begin{aligned}
\Delta_{h} \tilde{u}_{h}(x, y) & =M_{h}(\varphi,(x, y)) & & \left((x, y) \in \Omega_{h}\right) \\
\tilde{u}_{h}(x, y) & =0 & & \left((x, y) \in \Gamma_{h}\right)
\end{aligned}
$$

with some typical (averaging) integral operator $M_{h}$, it is not astonishing that the sharpness of the inequalities (11) and (15) each time depends on the same properties of $G_{h}(\xi, \eta)$. In other words, both logarithm factors originate form the unboundedness of the discrete Green function in the $l^{\infty}$-norm. 


\section{References}

[1] Büttgenbach, B., Esser, H., Lüttgens, G. and R. J. Nessel: A sharp error estimate for the numerical solution of a Dirichlet problem for the Poisson equation. J. Comput. Appl. Math. 44 (1992), 331 - 337.

[2] Büttgenbach, B., Esser, H. and R. J. Nessel: On the comparison of error bounds for finite difference schemes. Numer. Math. 64 (1993), 477 - 486.

[3] Büttgenbach, B., Lünebach, P., Lüttgens, G. and R. J. Nessel: Error bounds for compound quadrature rules in the space of Riemann integrable functions. In: Constructive Theary of Functions '91. Proc. Conf. Varna 1991 (eds.: K. G. Ivanov, P. P. Petrushev and Bl. Sendov). Sofia: Bulg. Acad. Sci. 1992, pp. $19-29$.

[4] Ciarlet, P. G.: Basic error estimates for elliptic problems. In: Handbook of Numerical Analysis. Vol. II: Finite Element Methods, Part 1 (eds.: P. G. Ciarlet and J. L. Lions). Amsterdam: North-Holland Publ. Comp. 1991, pp. 17 - 351.

[5] Dickmeis, W., Nessel, R. J. and E. van Wickeren: Quantitative extensions of the uniform boundedness principle. Jahresber. Dt. Math. Verein. 89 (1987), 105 - 134.

[6] Esser, H.: Stabilitätsungleichungen für Diskretisierungen von Randwertaufgaben gewöhnlicher Differentialgleichungen. Numer. Math. 28 (1977), 69 - 100.

[7] Esser, H., Kirchhoff, N., Lüttgens, G. and R. J. Nessel: On some properties of the $\tau$ modulus. World. Sci. Ser. Appl. Anal. (in print).

[8] Hackbusch, W.: Theorie und Numerik elliptischer Differentialgleichungen: Stuttgart: Teubner 1986 .

[9] Haverkamp, R.: Eine Aussage zur $L_{\infty}$-Stabilität und zur genauen Konvergenzordnung der $H_{0}^{1}$-Projektionen. Numer. Math. 44 (1984), 393 - 405.

[10] Isaacson, E. and H. B. Keller: Analyse numerischer Verfahren: Zürich: Harri Deutsch 1973.

[11] Lüttgens, G.: Scharfe Fehlerabschätzungen für die numerische Approximation von Lösungen elliptischer Differentialgleichungen. Dissertation. Aachen: Rhein.-Westfäl. Techn. Hochschule 1993.

[12] Michlin, S. G. and Ch. L. Smolizki: Näherungsmethoden zur Lösung von Differential- und Integralgleichungen (Mathematik für Technische Hochschulen: Vol. 10). Leipzig: B. G. Teubner Verlagsges. 1969.

[13] Nitsche, J.: $L_{\infty}$-convergence of finite element approximations. In: Mathematical Aspects of Finite Element Methods. Proc. Conf. Rome 1975 (eds.: I. Galligani and E. Magenes). Berlin: Springer-Verlag 1977, pp. 261 - 274.

[14] Sendov, Bl. and V. A.Popov: The Averaged Moduli of Smoothness. New York: Wiley 1988.

Received 24.02.1994 